\title{
Dual band low loss metamaterial structure at millimetre wave band
}

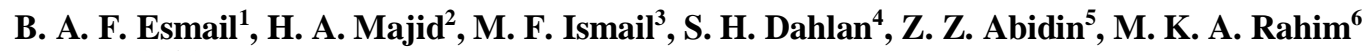 \\ 1,2,4,5 Applied Electromagnetics Center, Universiti Tun Hussein Onn Malaysia, Malaysia \\ ${ }^{3}$ Centre for Diploma Studies, Universiti Tun Hussein Onn Malaysia, Malaysia \\ ${ }^{6}$ Faculty of Electrical Engineering, Universiti Teknologi Malaysia, Malaysia
}

\begin{tabular}{l}
\hline Article Info \\
\hline Article history: \\
Received Oct 1, 2018 \\
Revised Dec 10, 2018 \\
Accepted Jan 25, 2019 \\
\hline
\end{tabular}

Keywords:

Losses

Metamaterials (MMs)

mm-wave

Negative refractive index

\begin{abstract}
In this paper, the Dual band modified split square resonator (MSSR) metamaterial (MM) structure was designed and numerically investigated at millimetre wave (mm-Wave) frequency range. The proposed structure operated at two resonance frequencies $28 \mathrm{GHz}$ and $32.54 \mathrm{GHz}$. The dualband behaviour of the proposed structure because of the self and mutual coupling between two metallic squares of the structure. Furthermore, The MSRR structure performed very well at both resonance frequencies by providing high transmission coefficient, S21, with a loss of $-0.3 \mathrm{~dB}(0.97$ linear scale) at lower resonance frequency $28 \mathrm{GHz}$ and $-0.18 \mathrm{~dB}(0.98$ linear scale) at upper resonance frequency $32.54 \mathrm{GHz}$. In this regard, the numerical simulation was conducted to optimize the MSSR structure in such a way that the ratio of effective inductance-to-capacitance $(\mathrm{L} / \mathrm{C})$ was raised. As a result, the inherent MM losses were reduced. The robust retrieval algorithm was utilized to reconstruct the refractive index, effective permittivity, and effective permeability and to verify the left-hand property of the proposed structure. The simulation results showed that the MSSR unit cell introduces two regions of the negative refractive index below the lower resonance frequency, $28 \mathrm{GHz}$ and above the upper resonance frequency, $32.54 \mathrm{GHz}$.
\end{abstract}

Copyright $(0) 2019$ Institute of Advanced Engineering and Science. All rights reserved.

\section{Corresponding Author:}

Huda Bin A Majid,

Faculty of Engineering Technology,

Universiti Tun Hussein Onn Malaysia,

86400 Parit Raja, Johor, Malaysia.

Email: mhuda@uthm.edu.my

\section{INTRODUCTION}

Since the first experimental validation of the metamaterials (MMs) in 1998 [1], these artificial materials have attracted a lot of attention within the scientific community for different frequency bands applications [2]. MMs are defined as an artificial periodic structure with unique electromagnetic characteristics not found in nature. The base theory of these materials is introduced by Russian scientist veslago in 1967 [3]. The unusual electromagnetic characteristics of the MMs enable a lot of practical applications such as using the MMs for shielding, clocking, transfer power, enhance antenna performance, superlens, resolution imaging enhancers [4-9].

The design of low loss MMs structures in microwave frequent range and mm-Wave frequency range is a very important issue which needs to be addressed in the field of electromagnetic research. Furthermore, the design of multi-band MMs had also been investigated in the literature [10, 11]. In [12] a dual band of the U-shaped resonator is investigated with the help of on different metallic conductivity. Multi-band periodic square metallic layer with T-Shaped gap operating at terahertz band has been studied and investigated in [13]. 
In recent times, the inherent loss in the MMs structures becomes a great challenge, which in turn limits the MMs' potential applications and late enable MMs based devices, especially at high frequencies range such as mm-Wave frequency range [14]. These losses are increased when the frequency is pushed to high frequency range [15]. The MMs losses are induced by two components which are ohmic losses (i.e. the dissipation energy due to the intrinsic ohmic losses of the conducting layer of the MM structure) and radiation losses (i.e. MM structure scatters the electromagnetic wave away from the incident wave) [16]. Generally, the transmission coefficient $\left(\mathrm{S}_{21}\right)$ is used to measure the losses in the MM structures. The near zero value ( $\mathrm{dB}$ scale) and near unity (linear scale) of $S_{21}$ represent low loss behavior [17]. In the literature, several techniques have been proposed to reduce the inherent MMs losses, where the most of these structures offer single-band low loss behavior [18-22]. The tailoring geometry can reduce the losses of the structure by avoiding the corners and sharp edges [18]. Several methods have been proposed to reduce the losses by canceling the radiation losses [19] and by using electromagnetically induced transparency (EIT) phenomenon $[20,21]$. Also, using high inductance-to-capacitance ratio (L/C) to mitigate the losses of the MM structure [22]. On the other hand, in the literature, few reports have been studied the multi-band low loss MM structures at microwave frequency range [23-24].

In this paper, MSSR structure is proposed to operate at mm-Wave with dual-band characteristics. This structure operates at $28 \mathrm{GHz}$ and $23.54 \mathrm{GHz}$. The dual-band observed for the proposed unit cell due to the self and mutual coupling between two split squares of the structure. Moreover, the MM structure introduces a low loss at two resonance frequency. In this regard, the ratio of effective inductance-tocapacitance $(\mathrm{L} / \mathrm{C})$ should be increased by optimizing the parameters of the proposed structure during the numerical simulation. As a result, the inherent MM losses are reduced. The constitutive parameters are retrieved using a well-known algorithm to verify the left-hand property of the proposed structure.

\section{DESIGN OF THE MM STRUCTURE}

The geometry of the proposed MSSR structure and the structural parameters of the simulation setup of the single MSSR unit cell are depicted in Figure 1 (a) and (b). The two split squares are printed on the front side of the Rogers RT5880 with relative permittivity 2.2, tangent-loss of 0.0009 , and a thickness of $0.381 \mathrm{~mm}$. The material of the metallic MSSR is the lossy metal copper with an electric conductivity of $\sigma=$ $5.8 \times 10^{\wedge} 7 \mathrm{~S} / \mathrm{m}$ and the thickness of $0.035 \mathrm{~mm}$. the simulation setup of the MSSR is shown in Figure 1(b) with ax $=2.2 \mathrm{~mm}$, ay=2.2 $\mathrm{mm}$ and az $=3 \mathrm{~mm}$. the geometry specifications of the proposed structure are tabulated in Table 1. MM structures are considered as an LC circuit because of their small size in comparison with operating wavelength. The MSSR unit cell is much smaller than the operating wavelength $(<\lambda / 4)$ thereby it represents by LC circuit model. Figure 1(c) displays the equivalent circuit model of the MSSR. The squares loops of the MSSR structure induce inductive effect, while the gaps of the structure induce the capacitive effect. These parameters can control the resonance characteristic of the MM structure. The equivalent circuit is composed of fourteen self-inductances and six capacitances. As shown In Figure 1(a), the MSSR structure is a symmetry structure regarding both $\mathrm{x}$ - and $\mathrm{y}$-axes. The vertically incident electromagnetic wave induces a current on the metallic layer which introduces self-inductances in each conducting strip. On the other hand, the gaps of the structure act as capacitances.

Table 1. The geometry specifications of the proposed MSSR structure

\begin{tabular}{cc}
\hline Parameters & Value $(\mathrm{mm})$ \\
\hline X & 2.2 \\
Y & 2.2 \\
L & 0.55 \\
L1 & 0.65 \\
L2 & 0.3 \\
U & 0.2 \\
G & 0.35 \\
G1 & 0.2 \\
S & 0.28 \\
S1 & 0.32 \\
S2 & 0.47 \\
\hline
\end{tabular}

To induce the electromagnetic (EM) wave and to retrieve the constitutive parameters of the proposed structure, the electric field is allocated along the x-axis and the magnetic field is assigned with $y$ axis. The wave has been let through by opening the $\mathrm{z}$-direction. The unit cell is simulated in the time domain using CST Microwave Studio. 

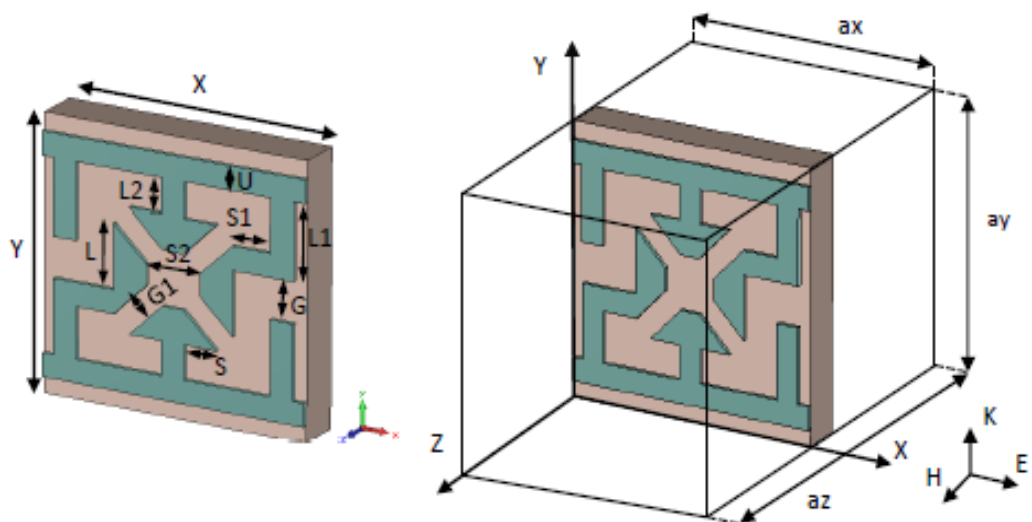

(a)

(b)

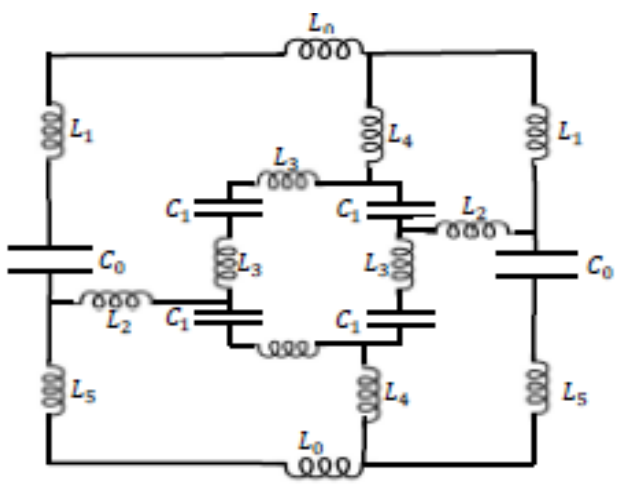

(c)

Figure 1. The proposed MSSR structure, a) schematic view, b) simulation setup, and c) equivalent circuit

\section{METHODOLOGY OF MM LOSS REDUCTION}

The main intention of this work is to design dual-band MM structure with low loses at mmWave frequency range. In our design, the conducting strips of the structure are increased because of the connection of the outer square strip to the inner one, thereby the self-inductance is increased. Moreover, the capacitance is also raised due to the increase in the structure's gaps. Hence, the resonance frequency is dropped swiftly $\mathrm{f}=1 / 2 \pi \sqrt{\mathrm{LC}}$. However, the inductance of the structure is excessively high in comparison with the capacitance. In the RLC circuit, the losses depend on the Q factor and the parameters of the circuit. The Q-factor is expressed as $\mathrm{Q}=(\mathrm{I} / \mathrm{R}) * \sqrt{ }(\mathrm{L} / \mathrm{C})$. The decrease in the capacitance and resistance or the increase in the inductance causes an increase in the Q-factor and thereby reducing the circuit losses. To reduce the resistance effect, a good conducting material with good conductivity should be chosen through the design. On the other hand, the geometric parameters of the structure are controlling the values of $\mathrm{L}$ and $\mathrm{C}$. By altering the geometric parameters of the structure, the values of $\mathrm{L}$ and $\mathrm{C}$ can be changed easily. Here, the losses reduction of the proposed structure can be obtained by exploiting the resonance circuit parameters $\mathrm{L}$ and $\mathrm{C}$. The strip length and width and the gaps of the structure are optimized through the simulation in such way that the L/C ratio is incremented and thereby the losses are extremely reduced. By implementing this method, the loss of the circular spiral split-ring resonators is mitigated [22].

\section{RESULTS AND ANALYSIS}

\subsection{S-parameters and MM losses}

The simulated reflection and transmission coefficients results of the proposed MSSR structure are shown in Figure 2. As can be seen, the MSSR structure operates at two resonance frequencies, $28 \mathrm{GHz}$ and $32.54 \mathrm{GHz}$. The electromagnetic wave totally passes through the unit cell within the bands of $27.57 \mathrm{GHz}$ to $28.42 \mathrm{GHz}$ and $32.35 \mathrm{GHz}$ to $32.8 \mathrm{GHz}$. Here, the simulation results achieve a return loss of $-28.8 \mathrm{~dB}$ and a bandwidth of $0.85 \mathrm{GHz}$ at $28 \mathrm{GHz}$ and a return loss of $-26.3 \mathrm{~dB}$ and a bandwidth of $0.45 \mathrm{GHz}$ at $32.54 \mathrm{GHz}$. Despite the drawback of the narrow bandwidth of MM, the MSSR achieves a good bandwidth at two resonance frequencies. 


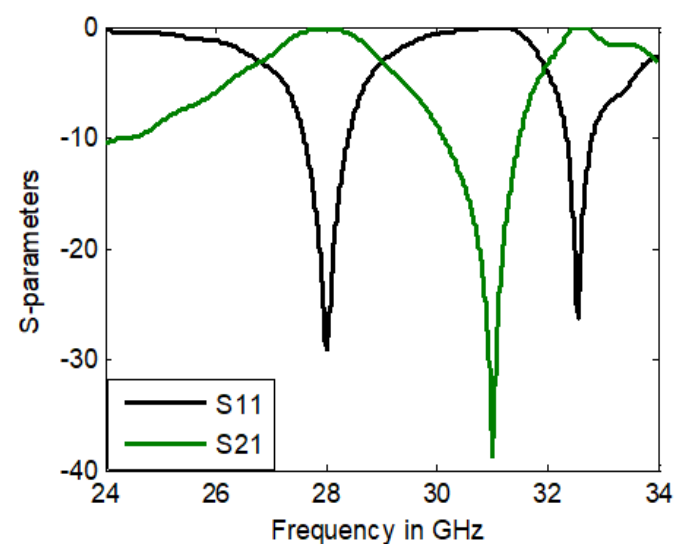

Figure 2. Transmission and reflection coefficients of the proposed MSSR structure

The inherent loss in the MM structures becomes a great challenge in a practical environment. These losses need to be studied more and addressed. The new MM structure with low loss behavior is in high demand especially at the high-frequency range where the inherent MM losses are raised when the frequency is increased. Figure 2 displays the simulated transmission coefficient result of the proposed MSSR structure. The MSSR structure introduces low loss behavior at the two resonance frequencies. At lower resonance frequency $28 \mathrm{GHz}$, the simulated transmission coefficient, which is the losses measure, is $-0.3 \mathrm{~dB}(0.97$ linear scale) and $-0.18 \mathrm{~dB}$ (0.98 linear scale) at upper resonance frequency $32.54 \mathrm{GHz}$. The proposed structure achieves almost full transmission at both resonance frequencies.

Figure 3 (a) and (b) shows the simulated surface current of the proposed MM structure at tow resonance frequencies, $28 \mathrm{GHz}$ and $32.54 \mathrm{GHz}$, respectively. For Figure 3(a), the surface current is concentered more at the top left corner and bottom right corner of the MSSR structure and this arrangement induces the lower resonance frequency which is $28 \mathrm{GHz}$. On the other hand, the surface current is focused more at the top right side which extends to the outer edge of the inner square and bottom left side of the MSSR structure and this arrangement contributes to exciting the upper resonance frequency which is 32.54 $\mathrm{GHz}$ as shown in Figure 3(b).

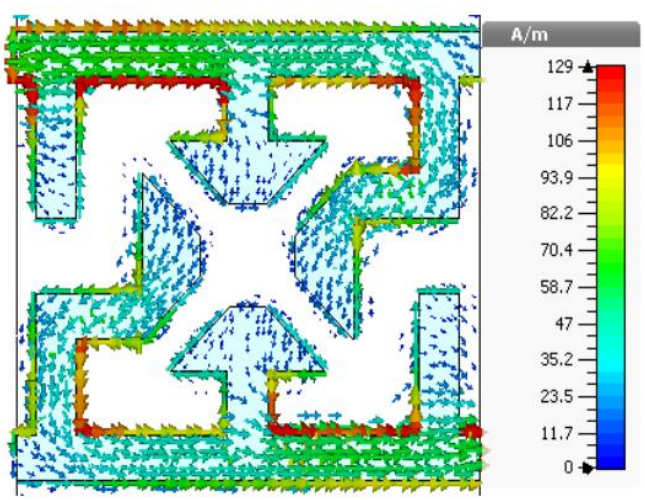

(a)

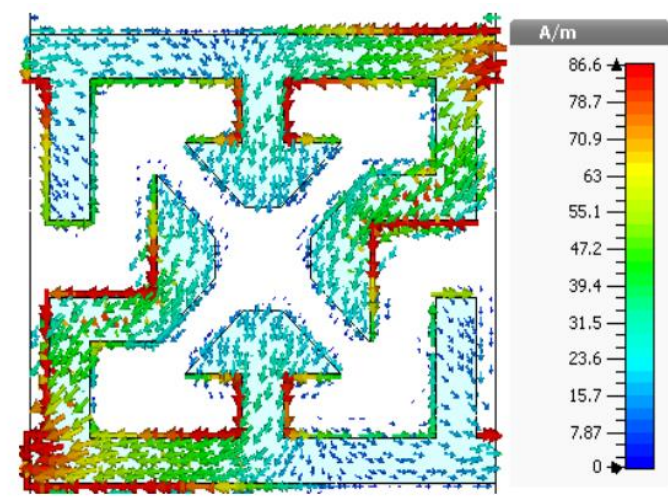

(b)

Figure 3. The simulated surface current of the MSSR structure a) at $28 \mathrm{GHz}$ b) at $32.54 \mathrm{GHz}$

\subsection{Constitutive parameters}

The effective-medium theory is used as the most common method to model the MMs structures. In this regard, some procedures and processes are implemented on the electric and magnetic fields of the MM structures. As a result, the constitutive parameters (effective permittivity, permeability, and refractive index) of the MM structure can be calculated. In this paper, the robust retrieval algorithm is utilized to reconstruct the effective constitutive parameters of the proposed structure [25]. To extract the desired constitutive parameters, the CST Microwave Studio-based time domain solver is used to determine the complex 
reflection and transmission properties of the proposed structure. After obtaining the complex S-parameters values the effective refractive index $n$ and impedance $z$ can be calculated. Subsequently, the permittivity and permeability can be calculated by using the $\varepsilon=n \times \mathrm{z}$ and $\mu=\mathrm{n} / \mathrm{z}$. The numerical simulation of the real and imaginary results of the refractive index is illustrated in Figure 4. The double negative nature of the MSSR structure is proven here by the negative refractive index, $\mathrm{n}$ at the dual-band $28 \mathrm{GHz}$ and $3.54 \mathrm{GHz}$ with values of -22 and -14 , respectively. The negative refractive index is discussed in the range of 25 to $34 \mathrm{GHz}$. It can be seen that the proposed structure introduces two regions of the negative refractive index below the resonance frequency at lower-frequency band $28 \mathrm{GHz}$ and above the resonance frequency at upper-frequency band $32.54 \mathrm{GHz}$. From the refractive index and impedance calculations, the effective permittivity and permeability are calculated using robust retrieve algorithm. Figures 5 and 6 show the real and imaginary effective permittivity and permeability of the proposed MSSR structure, respectively. It is noticeable that, effective permittivity and permeability are negative at the two resonance frequencies. Moreover, the proposed MSSR structure offers two regions of the negative permittivity below the lower resonance frequency $28 \mathrm{GHz}$ and above and below the upper resonance $32.54 \mathrm{GHz}$. On the other hand, MSSR structure provides two regions of the negative permeability below and above the two resonance frequencies $28 \mathrm{GHz}$ and $32.54 \mathrm{GHz}$.

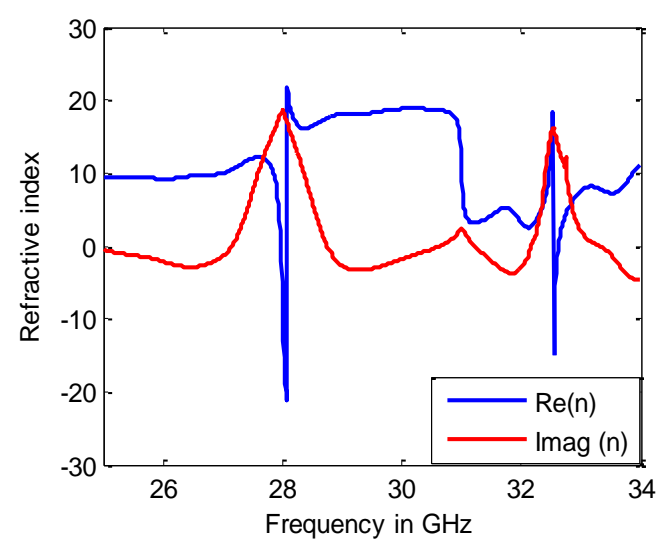

Figure 4. The simulated real and imaginary refractive index results of the proposed MSSR structure

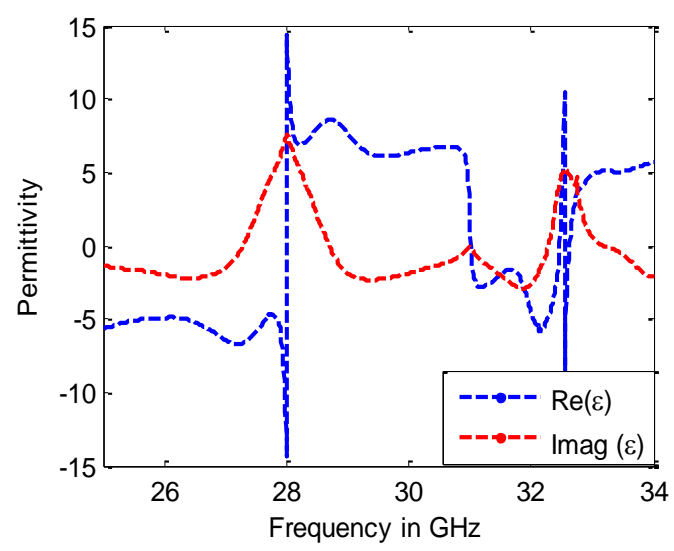

Figure 5. The simulated real and imaginary effective permittivity results of the proposed MSSR structure 


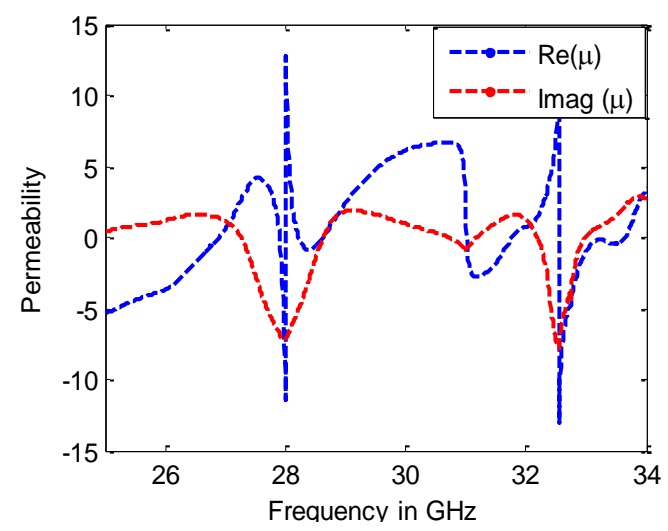

Figure 6. The simulated real and imaginary effective permeability results of the proposed MSSR structure

\section{CONCLUSION}

In summary, the low loss modified split square resonator (MSSR) structure with dual-band characteristics is studied and numerically investigated. The MSSR operates at mm-Wave frequency range with two resonance frequencies $28 \mathrm{GHz}$ and $32.54 \mathrm{GHz}$. The surface current at two resonance frequencies is presented and discussed to explain the dual-band characteristics. Furthermore, the low loss behaviour of the proposed structure is discussed at both resonance frequencies. The losses reduction of the proposed structure can be obtained by exploiting the resonance circuit parameters $\mathrm{L}$ and $\mathrm{C}$. The strip length and width and the gaps of the structure are optimized through the simulation in such way that the inductance-to-capacitance (L/C) ratio is incremented. As a result, the inherent MM losses are extremely reduced. The constitutive parameters are reconstructed using robust retrieve algorithm. The double negative nature of the proposed structure is verified by the negative refractive index at both resonance frequencies. The MSSR unit cell provides two regions of the negative refractive index below $28 \mathrm{GHz}$ and above $32.54 \mathrm{GHz}$. For the permittivity, the two negative regions are below $28 \mathrm{GHz}$ and above and below $32.54 \mathrm{GHz}$ while the two regions of the negative permeability are below and above both resonance frequencies $28 \mathrm{GHz}$ and 32.54 $\mathrm{GHz}$. The proposed structure has been investigated through the simulation. This study recommends as a future work test the same structure through the fabrication and measurement.

\section{ACKNOWLEDGEMENTS}

This work was supported by Ministry of Higher Education (MOHE), Faculty of Engineering Technology, Research Center of Applied Electromagnetics, Research Management Centre, Universiti Tun Hussein Onn Malaysia (UTHM) under Grant (Vote No: TIER1 U923 / GPPS U735 / FRGS 1614).

\section{REFERENCES}

[1] J. B. Pendry, et al. "Low frequency plasmons in thin wire structures," Journal of Physics: Condensed Matter, vol/issue: 10(22), pp. 4785, 1998.

[2] A. Ennajih, et al., "A New Dual Band Printed Metamaterial Antenna for RFID Reader Applications," International Journal of Electrical and Computer Engineering (IJECE), vol/issue: 7(6), pp. 3507-3514, 2017.

[3] V. G. Veselago, "The electrodynamics of substances with simultaneously negative values of and $\mu$," Soviet physics uspekhi, vol/issue: 10(4), pp. 509, 1968.

[4] G. Ababei, et al. "Left-handed metastructures with selective frequency transmission window for gigahertz shielding applications," Journal of Applied Physics, vol/issue: 117(17), pp. 17A502, 2015.

[5] S. Yang, et al. "From flexible and stretchable meta-atom to metamaterial: A wearable microwave meta-skin with tunable frequency selective and cloaking effects," Scientific reports, vol. 6, pp. 21921, 2016.

[6] K. Sun, et al., "An overview of metamaterials and their achievements in wireless power transfer," Journal of Materials Chemistry C, vol/issue: 6(12), pp. 2925-2943, 2018.

[7] S. I. Rosaline, et al., "Metamaterial-Inspired Split Ring Monopole Antenna for WLAN Applications," Applied Computational Electromagnetics Society Journal, vol/issue: 33(2), 2018.

[8] B. Majumder, et al. "A Zero Index Based Meta-Lens Loaded Wideband Directive Antenna Combined with Reactive Impedance Surface," IEEE Access, vol. 6, pp. 28746-28754, 2018.

[9] I. Escorcia, et al., "Terahertz metamaterial absorbers implemented in CMOS technology for imaging applications: scaling to large format focal plane arrays," IEEE Journal of Selected Topics in Quantum Electronics, vol/issue: 23(4), 2017. 
[10] B. X. Wang, "Single-patterned metamaterial structure enabling multi-band perfect absorption," Plasmonics, vol/issue: 12(1), pp. 95-102, 2017.

[11] S. S. Islam, et al., "The design and analysis of a novel split-H-shaped metamaterial for multi-band microwave applications," Materials, vol/issue: 7(7), pp. 4994-5011, 2014.

[12] B. X. Wang, et al., "Design of a novel dual-band terahertz metamaterial absorber," Plasmonics, vol/issue: 11(2), pp. 523-530, 2016.

[13] H. Y. Meng, et al., "A Simple Design of a Multi-Band Terahertz Metamaterial Absorber Based on Periodic Square Metallic Layer with T-Shaped Gap," Plasmonics, vol/issue: 13(1), pp. 269-274, 2018.

[14] Z. Wei, et al. "Analogue electromagnetically induced transparency based on low-loss metamaterial and its application in nanosensor and slow-light device," Plasmonics, vol/issue: 12(3), pp. 641-647, 2017.

[15] B. A. F Esmail, et al., "Novel Metamaterial Structures with Low Loss at Millimeter Wave Frequency Range," Indonesian Journal of Electrical Engineering and Computer Science, vol/issue: 10(2), pp. 641-647, 2018.

[16] A. V. Zayats, et al., "Active plasmonics and tuneable plasmonic metamaterials," John Wiley \& Sons, vol. 8, 2013.

[17] B. A. F. Esmail, et al., "Refractive index reconfigurable metamaterial structure at $28 \mathrm{GHz}$ frequency range," Microwave Conference (APMC), 2017 IEEE Asia Pacific, pp. 1325-1328, 2017.

[18] D. Ö. Güney, et al., "Reducing ohmic losses in metamaterials by geometric tailoring," Physical Review B, vol/issue: 80(12), pp. 125129, 2009.

[19] L. Zhu, et al., "Low-loss magnetic metamaterial at THz frequencies by suppressing radiation losses," IEEE Transactions on Terahertz Science and Technology, vol/issue: 3(6), pp. 805-811, 2013.

[20] X. JunáHe and C. HuiáZhao, "A low-loss electromagnetically induced transparency (EIT) metamaterial based on coupling between electric and toroidal dipoles," RSC Advances, vol/issue: 7(88), pp. 55897-55904, 2017.

[21] Y. Tian, et al., "Low-loss planar metamaterials electromagnetically induced transparency for sensitive refractive index sensing," Journal of Physics D: Applied Physics, vol/issue: 50(40), pp. 405105, 2017.

[22] T. Shaw and D. Mitra, "Design of miniaturized, low-loss and flexible multi-band metamaterial for microwave application," Applied Physics A, vol/issue: 124(4), pp. 348, 2018.

[23] C. Sabah, "Multiband metamaterials based on multiple concentric open-ring resonators topology," IEEE Journal of Selected Topics in Quantum Electronics, vol/issue: 19(1), pp. 8500808-8500808, 2013.

[24] C. Zhu, et al., "Low-loss triangular left-and right-handed metamaterials," Electronics letters, vol/issue: 47(1), pp. 12-14, 2011.

[25] X. Chen, et al., "Robust method to retrieve the constitutive effective parameters of metamaterials," Physical Review E, vol/issue: 70(1), pp. 016608, 2004.

\section{BIOGRAPHIES OF AUTHORS}
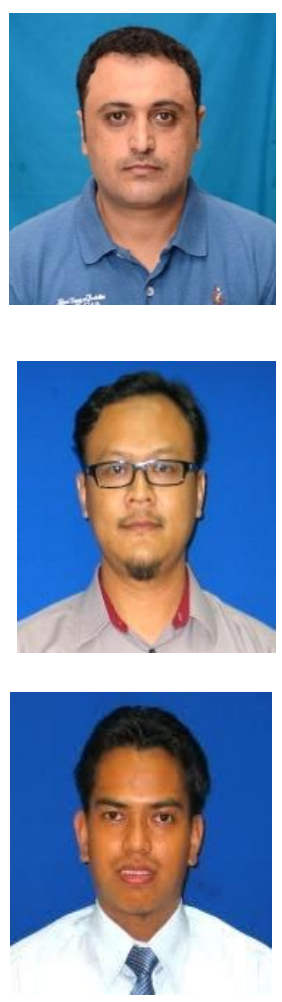

Bashar Ali Esmail received the B Eng. degree in Electrical Engineering (Telecommunications) with honours from Ibb University-Yemen, in 2008. He then obtained his M.Eng (Electrical Engineering) in 2016, at Universiti Tun Hussein Onn Malaysia. He is currently a on going PhD in Electrical Engineering at Faculty of Electrical and Electronic Engineering Universiti Tun Hussein Onn Malaysia. His research interest includes the areas of design of metamaterials structure, milimter wave antenna and reconfigurable antenna.

Huda A Majid received the B Eng. degree in Electrical Engineering (Telecommunication) from Universiti Teknologi Malaysia, in 2007. He then obtained his M.Eng in 2010 and PhD degrees in Electrical Engineering in 2013, at Universiti Teknologi Malaysia. He is currently a lecturer in the Department of Electrical Engineering Technology, Faculty of Engineering Technology, Universiti Tun Hussein Onn Malaysia. His research interest includes the areas of design of microstrip antennas, small antennas, Reconfigurable antennas, metamaterials structure, metalaterial antennas and millimeter wave antennas. He has published over 100 articles in journals and conference papers.

Muhammad Faizal Ismail received the $\mathrm{B}$ Eng. degree in Electrical Engineering (Telecommunications) with honours from Universiti Teknologi Malaysia, in 2009. He then obtained his M.Eng (Electrical Engineering) in 2011, at Universiti Teknologi Malaysia and also as ongoing PHD at same university. His research interest includes the areas of design of electromagnetic band gap (EBG) structure, microstrip antenna and pattern reconfigurable EBGs antenna. 

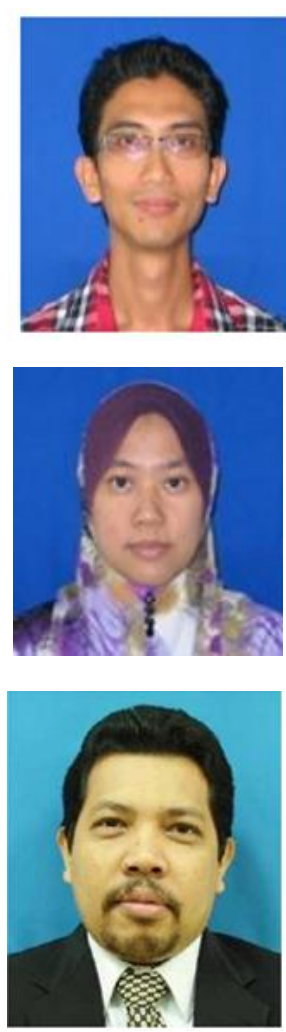

Samsul Haimi Bin Dahlan received the Bachelor's degree in Engineering from the Universiti Kebangsaan of Malaysia, Bangi, Malaysia, in 1999, the Master's degree in engineering from Universiti Teknologi Malaysia, Johor Bahru, Malaysia, in 2005, and the Ph.D. degree from Universite' de Rennes 1, Rennes, France, in 2012. He is currently the Head of the Research Center for Applied Electromagnetics, Universiti Tun Hussein Onn Malaysia (UTHM), Batu Pahat, Malaysia. His research interests include EMC, electromagnetic shielding, bioelectromagnetics, microwave devices, advanced antenna design, material characterization, and computational electromagnetics. Dr. Samsul has published many conference proceedings as well as journal papers in local and international journals.

Zuhairiah Zainal Abidin was born in Kuala Lumpur, Malaysia, in 1978. She received the B.Eng.from the Universiti Teknologi Malaysia, in 2001, the M. Eng from the Kolej Universiti Tun Hussein Onn Malaysia, Johor, Malaysia, in 2003, and Ph.D. degree from Bradford University, U.K in 2011. Currently, she was a Senior Lecturer at Universiti Tun Hussein Onn Malaysia. Her current research interests include MIMO antenna design, electromagnetic bandgap (EBG) for wireless and mobile systems and high speed digital circuits and wearable antennas.

Mohamad Kamal A Rahim received the B Eng. degree in Electrical and Electronic Engineering from University of Strathclyde, UK, in 1987. In 1989, he joined the Department of Communication Engineering, Faculty of Electrical Engineering Universiti Teknologi Malaysia Kuala Lumpur as an Assistant Lecturer A. He obtained his M.Eng Science from University of New South Wales Australia in 1992 and PhD degrees in Electrical Engineering from University of Birmingham UK in 2003. He is the Professor in RF and Antenna at Faculty of Electrical Engineering Universiti Teknologi Malaysia. His research interest includes the areas of design of dielectric resonator antennas, microstrip antennas, small antennas, microwave sensors, RFID antennas for readers and tags, Multi-function antennas, microwave cicuits, EBG, artificial magnetic conductors, metamaterials, phased array antennas, computer aided design for antennas and design of millimeter frequency antennas. He has published over 200 articles in journals and conference papers. 